\begin{tabular}{|c|l|}
\hline Title & Coherent Shear Phonon Generation and Detection with UItrashort Optical Pulses \\
\hline Author(s) & Matsuda, O.; Wright, O. B.; Hurley, D. H.; Gusev, V. E.; Shimizu, K. \\
\hline Citation & $\begin{array}{l}\text { Physical Review Letters, 93(9), 095501 } \\
\text { https://doi.org/40.1103/PhysRevLett.93.095501 }\end{array}$ \\
\hline Issue Date & 2004 \\
\hline Doc URL & http://hdl.handle.net/2115/14637 \\
\hline Rights & Copyright $\odot 2004$ A merican Physical Society \\
\hline Type & article \\
\hline File Information & PRL93-9pdf.pdf \\
\hline
\end{tabular}

Instructions for use 


\title{
Coherent Shear Phonon Generation and Detection with Ultrashort Optical Pulses
}

\author{
O. Matsuda, ${ }^{1, *}$ O. B. Wright, ${ }^{1}$ D. H. Hurley, ${ }^{2}$ V. E. Gusev, ${ }^{3}$ and K. Shimizu ${ }^{4}$ \\ ${ }^{1}$ Department of Applied Physics, Graduate School of Engineering, Hokkaido University, Sapporo 060-8628, Japan \\ ${ }^{2}$ Idaho National Engineering and Environmental Laboratory, Idaho Falls, Idaho 83415-2209, USA \\ ${ }^{3}$ Université du Maine, Avenue O. Messiaen, 72085 Le Mans, France \\ ${ }^{4}$ University Chemical Laboratory, Keio University, Yokohama, Kanagawa 223-8521, Japan
}

(Received 5 April 2004; published 24 August 2004)

\begin{abstract}
Using an optical technique we generate and detect picosecond shear and quasishear coherent acoustic phonon pulses in the time domain. Thermoelastic and piezoelectric generation are directly achieved by breaking the sample lateral symmetry using crystalline anisotropy. We demonstrate efficient detection in isotropic and anisotropic media with various optical incidence geometries.
\end{abstract}

DOI: 10.1103/PhysRevLett.93.095501

By shaking atoms one may assess interatomic bond strengths and the integrity of crystal lattices. In particular this may be achieved by high-frequency phonon excitation and detection, providing a wealth of information on the elastic properties of solids on nanometer and atomic length scales owing to the enhancement in scattering when the phonon wavelength is of the same order as the structure under investigation. This field of research, initially driven by terahertz phonon measurements involving superconducting tunnel junctions, heat pulses, phonon-induced fluorescence, and Raman or Brillouin scattering [1,2], has more recently been supplemented with ultrafast optical techniques in the time domain. In particular, such impulsive optical generation and delayedtime optical probe detection at surfaces permits the use of propagating $\mathrm{GHz}-\mathrm{THz}$ phonon pulses to acoustically inspect the interior of nanostructures [3-10]. Acoustic phonon generation with ultrashort optical pulses is enabled by a variety of mechanisms, such as themoelasticity [3-9], deformation potential coupling [10,11], or screening of electric fields combined with piezoelectricity $[12,13]$. The respective excitation of thermal phonons, carriers, or (rapid changes in) screening potential in an opaque material produce an initial stressed near-surface region whose size in the lateral direction ( $\gtrsim 1 \mu \mathrm{m}$ ) depends on the optical spot diameter and in the depth direction ( $\lesssim$ $100 \mathrm{~nm}$ ) on optical absorption, carrier diffusion or builtin electric field localization. Phonon detection is achieved through the photoelastic effect or surface displacement when the phonon pulse returns to the same point on the surface after scattering within a short distance. In this case, with isotropic media or symmetrically cut crystals, the constraints of symmetry imply that one only excites longitudinal acoustic phonons in the depth direction.

Such longitudinal acoustic phonon experiments have lead to picosecond time-scale studies involving as diverse a range of subjects as ultrashort time-scale carrier diffusion in metals and semiconductors [5,9,10], highfrequency ultrasonic attenuation in crystals and glasses $[14,15]$, phonon generation and detection in semiconduc-
PACS numbers: 63.20.Dj, 43.35.+d, 78.20.Hp, 78.47.+p

tor quantum wells and superlattices $[6,12,16]$, and soliton propagation and their coupling to two-level systems in ruby $[7,17]$. In spite of these successes, these experiments only address one of the three acoustic polarizations. To match the impressive capabilities of Brillouin and Raman scattering techniques one would naturally wish to simultaneously detect all three. For the example of an isotropic solid, where the two shear modes are degenerate, this gives access to the shear elasticity tensor component $c_{44}$ as well as the longitudinal component $c_{11}$. Moreover, the shear wave velocity $v_{t}$ is lower, leading to a shorter wavelength for a given frequency that is advantageous for nanoscale probing. However, tracking coherent picosecond phonon propagation and detection in the ultrashort time domain has so far been limited by the difficulty to directly generate such high-frequency shear phonons impulsively; this has so far only been achieved with nanosecond temporal resolution [18].

Motivated by our initial success in detecting shear phonon pulses indirectly produced by the (relatively inefficient) mode conversion of picosecond longitudinal phonon pulses at an isotropic-anisotropic interface [19], we extend the method to achieve the direct and efficient ultrafast optical excitation of shear phonon pulses in the anisotropic medium itself, using surfaces cut off-axis of the single crystals $\mathrm{Zn}$ and GaAs as representative examples with different phonon generation mechanisms. We also augment the possibilities for optical detection with a variety of probe incidence configurations.

Consider first the case of shear phonon generation through thermoelasticity alone, as in a typical metal. If the surface of an opaque material is heated by an ultrashort light pulse in the form of a plane electromagnetic wave, an acoustic pulse is launched in the depth direction through thermal expansion [3]. For arbitrarily cut anisotropic materials the allowed acoustic modes for this propagation direction (the $z$ axis) are not pure longitudinal or shear in general, and it is possible to generate shear strain components as a consequence of the broken symmetry. In particular, if the thermal expansion tensor is 
itself anisotropic a component of the initial shear stress can be generated directly, instantaneously and with optimum efficiency. To test this scenario we choose such an opaque substrate coated with a transparent isotropic film. This facilitates the photoelastic detection analysis in the film, which supports only pure shear or longitudinal polarizations. Recall that in any medium $z$-propagating strain has nonzero shear components $\eta_{4}=2 \eta_{y z}$ or $\eta_{5}=$ $2 \eta_{x z}$ and a longitudinal component $\eta_{3}=\eta_{z z}$. These modulate the permittivity tensor $\varepsilon$ of the isotropic film

$$
\Delta \varepsilon=\left(\begin{array}{ccc}
P_{12} \eta_{3} & 0 & P_{44} \eta_{5} \\
0 & P_{12} \eta_{3} & P_{44} \eta_{4} \\
P_{44} \eta_{5} & P_{44} \eta_{4} & P_{11} \eta_{3}
\end{array}\right)
$$

where $P_{11}, P_{12}$, and $P_{44}=\left(P_{11}-P_{12}\right) / 2$ are photoelastic tensor components [20]. Since the coupling of $\Delta \varepsilon$ to shear strain is off diagonal and involves the suffix $z$, the corresponding induced change in optical reflectivity $R$ depends only on the $z$ component of the probe light electric field. Therefore, $p$-polarized probe light at oblique incidence must be used to detect shear waves in the film. A detailed analysis reveals the following, assuming the $x$ axis (Fig. 1) to lie within the plane of incidence [21,22]: (i) only $\eta_{4}$ shear strain can be detected; (ii) $\eta_{4}$ scatters $s$-polarized to $p$-polarized light and vice versa; (iii) a first order change in $R\left(\delta R \propto \eta_{4}\right)$ can be obtained only by allowing the incident and reflected light to interfere (otherwise $\delta R \propto \eta_{4}^{2}$ ).

The analysis for optical strain detection in the anisotropic substrate is more complex. By reference to an offaxis cut of a hexagonal crystal we outline the main principles. Assuming that the cut is obtained by a single rotation about the $x$ axis off the $c$ axis we find [20]

$$
\Delta \varepsilon=\left(\begin{array}{ccc}
P_{1 i} \eta_{i} & P_{56} \eta_{5} & P_{55} \eta_{5} \\
P_{56} \eta_{5} & P_{2 i} \eta_{i} & P_{i 4} \eta_{i} \\
P_{55} \eta_{5} & P_{i 4} \eta_{i} & P_{3 i} \eta_{i}
\end{array}\right),
$$

where $i=3,4$ is a summation index. The shear strain now perturbs the diagonal components of $\Delta \varepsilon$. Therefore no particular conditions on the nature of the probe light are posed in this case.

For the substrate we use a zinc single crystal of the hexagonal system $(6 / \mathrm{mmm})$, that exhibits thermoelastic phonon generation through a highly anisotropic uniaxial thermal expansion. (The $c$-axis thermal expansion coefficient is $6.4 \times 10^{-5} \mathrm{~K}^{-1}$ and that perpendicular is $1.3 \times$ $10^{-5} \mathrm{~K}^{-1}$ [20].) The $\mathrm{Zn}$ is cut with a surface $\phi=24^{\circ}$ off the (0001) plane, close to the angle $28.8^{\circ}$ for maximum shear strain generation following a temperature rise $(\sim$ $10 \mathrm{~K}$ here). The surface is trimmed with a diamond knife using an ultramicrotome to obtain a highly flat and plastic-deformation free region $(1 \mathrm{~mm} \times 1 \mathrm{~mm})$, thus avoiding a disordered boundary layer; atomic force mi- croscopy gives a surface roughness less than $1 \mathrm{~nm}$. An amorphous silica film of nominal thickness $1.1 \mu \mathrm{m}$ is then rf sputtered on the $\mathrm{Zn}$ surface.

A mode-locked Ti:sapphire laser beam of pulse duration $\sim 400 \mathrm{fs}$, repetition rate $76 \mathrm{MHz}$ and wavelength $814 \mathrm{~nm}$ is focused onto the $\mathrm{SiO}_{2}-\mathrm{Zn}$ interface with optical spot diameter $\sim 60 \mu \mathrm{m}$ and fluence $\sim 0.05 \mathrm{~mJ} \mathrm{~cm}^{-2}$. For our $\mathrm{Zn}$ crystal orientation the generated $z$-propagating acoustic modes correspond to quasilongitudinal, quasishear, and pure shear polarizations. Defining the $c$ axis to be contained in the $y z$ plane, only $\eta_{4}$ shear is generated. This strain component in $\mathrm{Zn}$ contributes most to the quasishear mode. Strains $\sim 5 \times 10^{-4}$ are produced.

For detection we focus time-delayed frequencydoubled probe light pulses (of wavelength $407 \mathrm{~nm}$ ) at $45^{\circ}$ incidence - with a similar fluence to that of the pump - to a $\sim 3$ times smaller spot area, and use a standard pump modulation technique. Four different optical polarization geometries are used (see Fig. 1): $p-u(s-u)$ denotes $p(s)$ polarized incident light and no analyzer, whereas 45- $s(45-p)$ denotes linearly polarized incidence characterized by a polarization axis at $45^{\circ}$ to the plane of incidence and an inserted analyzer for $s(p)$ polarized detection. Only 45- $s$ and 45- $p$ allow optical interference, thus enabling shear wave detection in the isotropic film.

Figure 1 shows the measured variation $\delta R(t) / R$, characterized by oscillations followed by echoes. We have subtracted a slowly varying background caused by temperature decay. Interpretation is facilitated by reference to a theoretical snapshot of the strain profile in the sample, shown at the bottom of Fig. 1 at delay time 100 ps without the static strain component, calculated from thermal expansion in the absence of diffusion or attenuation processes. Strain pulses in the $\mathrm{Zn}$ substrate and the $\mathrm{SiO}_{2}$ film are responsible for the echoes and the oscillations, respectively. We shall discuss the echoes first.

The echoes at $400 \mathrm{ps}$, seen in all four detection configurations, are due to the arrival of a (pure) longitudinal phonon pulse at the Zn substrate after reflection from the film top surface. The estimated longitudinal velocity $\boldsymbol{v}_{l} \approx$ $5.5 \mathrm{~km} \mathrm{~s}^{-1}$ in $\mathrm{SiO}_{2}$ is consistent with previous determinations for sputtered films. Part ( $\sim 24 \%$ ) of this phonon pulse is reflected back into the $\mathrm{SiO}_{2}$, and its second arrival at the $\mathrm{Zn}$ substrate produces inverted second echoes at $800 \mathrm{ps}$. The sign inversion of the strain is caused by the $\pi$ phase change at the top surface combined with the zero phase change at the $\mathrm{SiO}_{2}-\mathrm{Zn}$ interface (owing to the higher acoustic impedance of the $\mathrm{Zn}$ layer) [23].

The echoes near $700 \mathrm{ps}$ can be attributed to the arrival of the slower (pure) shear phonon pulse at the $\mathrm{Zn}$ substrate after reflection from the film top surface. The ratio of the estimated shear velocity $v_{t} \approx 3.2 \mathrm{~km} \mathrm{~s}^{-1}$ to $v_{l}$ is as expected [23]. In contrast to the case for the isotropic $\mathrm{SiO}_{2}$ film, the $\mathrm{Zn}$ substrate has nonvanishing photoelastic tensor components $P_{14}, P_{24}$, and $P_{34}$ that mediate the 

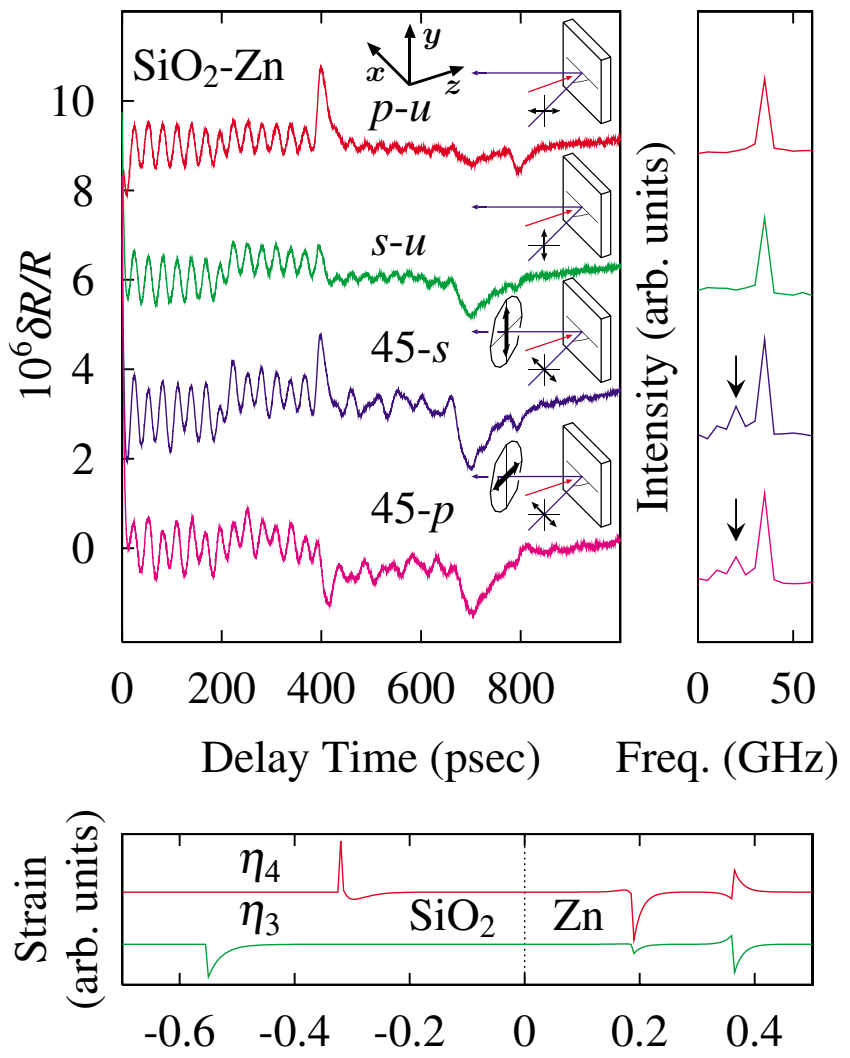

Distance from $\mathrm{SiO}_{2}-\mathrm{Zn}$ interface $(\mu \mathrm{m})$

FIG. 1 (color online). Transient relative reflectivity changes for a $1.1 \mu \mathrm{m}$ film of $a-\mathrm{SiO}_{2}$ on $\mathrm{Zn}$ and corresponding Fourier spectra for the first $200 \mathrm{ps}$. The pump beam (normally incident rays) and probe beam polarization configurations are shown in the insets. The arrows indicate the Fourier components for shear phonons. The lower graph shows a snapshot of the propagating strain components at 100 ps.

perturbation to the diagonal components of $\Delta \varepsilon$ by $\eta_{4}$ [see Eq. (2)]. The relative size of the echoes for the different phonon polarizations depends strongly on these components and on the detection configuration.

The temporal widths of the longitudinal and shear echoes are significantly broadened compared to the durations of 4 and 8 ps estimated under the oversimplified assumption that the strain pulse duration is given by the pump optical penetration depth $\sim 14 \mathrm{~nm}$ [24] divided by the quasilongitudinal or quasitransverse sound velocity of $\mathrm{Zn}\left(v_{q l}=3.6 \mathrm{~km} \mathrm{~s}^{-1}\right.$ and $v_{q t}=1.9 \mathrm{~km} \mathrm{~s}^{-1}$ at $\left.\phi=24^{\circ}\right)$. This difference might be attributable to the effects of ultrasonic attenuation in silica combined with ultrafast (anisotropic) nonequilibrium electron and thermal diffusion in $\mathrm{Zn}[5,9,25]$; strain pulse durations longer than $100 \mathrm{ps}$ have previously been observed in gold for similar optical penetration depths, for example [5].

The oscillations in the reflectivity are known to arise from the interference between the light reflected at the surface or interface and the light scattered at the prop- agating phonon pulses in the transparent film $[8,26]$. The oscillation frequency $f$ is related to the sound velocity $v$ and probe wavelength $\lambda$ through $f=2 n v \cos \theta / \lambda$, where $n$ is the refractive index of the film and $\theta$ is the angle of probe optical incidence therein. For the $p-u$ and $s-u$ configurations, that prohibit shear phonon detection in the isotropic film, only the single frequency $35.0 \mathrm{GHz}$ is observed, corresponding to the expected value for longitudinal phonon propagation (with $n=1.47, \lambda=407 \mathrm{~nm}$, and $\theta \approx 16^{\circ}$ from Snell's law). This is confirmed by examination of the Fourier spectra obtained for 0 to 200 ps (Fig. 1). Other features of these oscillations can also be explained: the small remnants persisting from 400 to $800 \mathrm{ps}$ are caused by the weak acoustic reflection from the $\mathrm{SiO}_{2}-\mathrm{Zn}$ interface, and the steps at 200 ps correspond to the arrival of the longitudinal acoustic pulse at the sample surface [8]. For the 45- $s$ and 45- $p$ configurations, that permit shear phonon detection in the film through $P_{44}$ in silica in Eq. (1), we indeed observe an extra frequency component at $20.0 \mathrm{GHz}$, evident not only in the time domain as a $30 \%$ modulation in the amplitude envelope but also in the corresponding Fourier spectra in Fig. 1. The frequency is as expected with $v=v_{t}=$ $3.2 \mathrm{~km} \mathrm{~s}^{-1}$. The slight decay in amplitude of the oscillations up to $400 \mathrm{ps}$ is consistent with the known ultrasonic attenuation in silica [25]. These findings, together with the observed echoes, provide conclusive evidence that we have achieved direct excitation and detection of picosecond shear phonon pulses.

In contrast to metals, semiconductors show a richer range of ultrafast phonon generation mechanisms $[10,27]$. Here we investigate the possibility of picosecond shear phonon generation in a single crystal of gallium arsenide of the cubic system $(\overline{4} 3 \mathrm{~m})$. The Si-doped GaAs (to $\sim 2 \times 10^{18} \mathrm{~cm}^{-3}$ ) is cut and prepared with a highly polished (411) surface. In contrast to the case for $\mathrm{Zn}$, physical quantities expressed by second rank tensors such as the permittivity or thermal expansion for GaAs are isotropic - the effects of anisotropy are only manifested in third rank tensors such as the piezoelectric tensor or fourth rank tensors such as the elastic constant or photoelastic tensors. Shear phonon generation by thermoelasticity or deformation potential coupling is, however, possible with off-axis crystal cuts since the initial hydrostatic stress should be resolved into the three $z$-propagating modes. Moreover, the lack of inversion symmetry in GaAs brings into play direct piezoelectric shear phonon generation through screening of electric fields by photoexcited carriers, that is enhanced by the sample doping. Since GaAs is semitransparent at infrared wavelengths, one can monitor quasishear phonon pulse generation and detection without using a transparent film.

Phonon generation is achieved with frequency-doubled pump light pulses of wavelength $405 \mathrm{~nm}$, focused onto the GaAs surface at normal incidence with an incident 


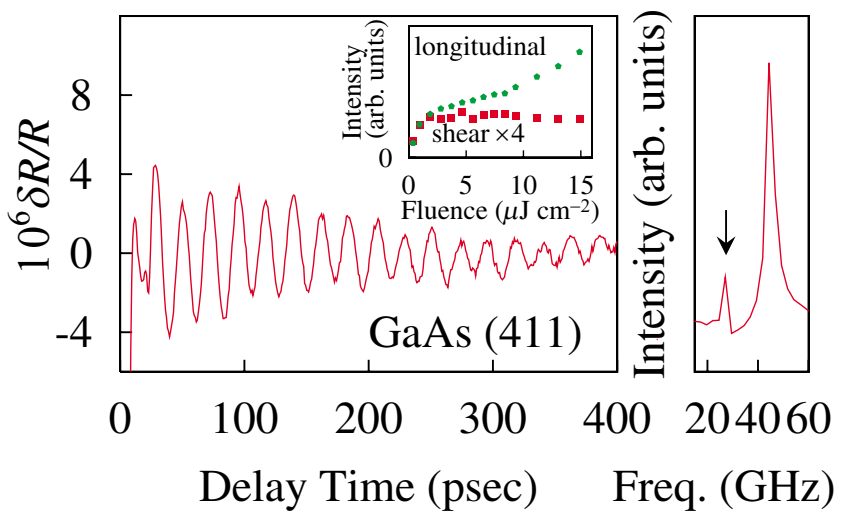

FIG. 2 (color online). Transient relative reflectivity change and its Fourier spectrum for GaAs (411). The arrow indicates the Fourier component for quasishear phonons. Inset: Fourier amplitudes as a function of pump fluence.

fluence up to $15 \mu \mathrm{J} \mathrm{cm}^{-2}$. Collinear linearly polarized probe light pulses of wavelength $810 \mathrm{~nm}$ are focused to the same point at normal incidence. The optical penetration depths of the pump and probe light are 17 and $790 \mathrm{~nm}$, respectively [28].

Typical experimental data at a pump fluence $\sim 5 \mu \mathrm{J} \mathrm{cm}^{-2}$ (and with the probe light polarization perpendicular to the expected shear displacement) is shown in Fig. 2, in which a slowly varying background due to the relaxation of excited carriers and thermal diffusion has been subtracted. The oscillations, having a similar origin as in the $\mathrm{SiO}_{2}-\mathrm{Zn}$ experiment but decaying owing to probe absorption, are composed of two distinct components; as shown by the Fourier spectra in Fig. 2, the frequencies are 45.0 and $27.4 \mathrm{GHz}$, corresponding to $v=4.98$ and $3.03 \mathrm{~km} \mathrm{~s}^{-1}$, respectively, according to $f=2 n v \cos \theta / \lambda$, with $n=3.66, \lambda=810 \mathrm{~nm}$, and $\theta=0^{\circ}$. These values are very close to the expected velocities of the quasilongitudinal mode $\left(4.97 \mathrm{~km} \mathrm{~s}^{-1}\right)$ and quasishear mode $\left(3.02 \mathrm{~km} \mathrm{~s}^{-1}\right)$ for $z$ propagation [28]. As required by symmetry considerations precluding its excitation, the pure shear mode $\left(3.24 \mathrm{~km} \mathrm{~s}^{-1}\right)$ is not observed.

The detection process can be explained by reference to Eq. (2). For cubic GaAs cut off-axis as in our case this equation is also valid. The pertinent strain components for $z$-directed propagation are $\eta_{3}$ and $\eta_{4} \simeq-0.34 \eta_{3}$ for the quasilongitudinal mode, and $\eta_{4}$ and $\eta_{3} \simeq 0.09 \eta_{4}$ for the quasishear mode. The absent pure shear mode is $\eta_{5}$. Diagonal perturbation by both the quasishear and quasilongitudinal modes enables optical detection irrespective of the angle of incidence or polarization.

The pump power dependences of the Fourier mode amplitudes (Fig. 2 inset) show striking nonlinear behavior including saturation. This suggests that piezoelectric coupling combined with ultrafast quenching of the local electric potential play a significant role in the generation, as previously noted at lower frequencies [18]; this mecha- nism is expected to be intensity independent for optical fluences sufficient for complete screening of the built-in electric field (in our case apparently at $\sim 5 \mu \mathrm{J} \mathrm{cm}^{-2}$ ). Further discussion is beyond the scope of this Letter.

In conclusion, we have demonstrated the generation and detection of shear and quasishear coherent acoustic phonon pulses with ultrashort optical pulses in the picosecond time domain. The generation is shown to be intricately tied to the symmetry properties of the tensors representing the relevant physical properties. Pure shear phonon pulses propagating in an isotropic material are detected with oblique optical incidence by an interference technique, whereas in anisotropic materials they are simply detected with normal incidence. This work should lead to the routine use of picosecond shear phonon pulses in studies indispensable for the evaluation of nanocrystalline structures, and help accelerate the development of nanoscale anisotropic engineering. Moreover, it should open the way to studies of picosecond shear motion at interfaces and ultrashort time-scale tribology.

*Electronic address: omatsuda@eng.hokudai.ac.jp

[1] R. Loudon, Adv. Phys. 50, 813 (2001).

[2] M. Wybourne et al., Rep. Prog. Phys. 51, 923 (1988).

[3] C. Thomsen et al., Phys. Rev. B 34, 4129 (1986).

[4] O. B. Wright et al., Phys. Rev. Lett. 69, 1668 (1992).

[5] O. B. Wright, Phys. Rev. B 49, 9985 (1994).

[6] B. Bonello et al., Ultrasonics 35, 223 (1997).

[7] H. Y. Hao et al., Phys. Rev. B 64, 064302 (2001).

[8] O. Matsuda et al., J. Opt. Soc. Am. B 19, 3028 (2002).

[9] T. Saito et al., Phys. Rev. B 67, 205421 (2003).

[10] O. B. Wright et al., Phys. Rev. B 64, 081202(R) (2001).

[11] M. D. Cummings et al., Appl. Phys. Lett. 79, 770 (2001).

[12] C. K. Sun et al., Phys. Rev. Lett. 84, 179 (2000).

[13] E. Makarona et al., Appl. Phys. Lett. 81, 2791 (2002).

[14] C. J. Morath et al., Phys. Rev. B 54, 203 (1996).

[15] W. Chen et al., Philos. Mag. B 70, 687 (1994).

[16] J. J. Baumberg et al., Phys. Rev. Lett. 78, 3358 (1997).

[17] O. L. Muskens et al., Phys. Rev. Lett. 92, 035503 (2004).

[18] S. A. Telenkov et al., J. Acoust. Soc. Am. 97, 1326 (1995).

[19] D. H. Hurley et al., Ultrasonics 38, 470 (2000).

[20] J.F. Nye, Physical Properties of Crystals (Oxford University Press, Oxford, 1957).

[21] O. Matsuda et al., Anal. Sci. 17, S216 (2001).

[22] O. Matsuda et al., Rev. Sci. Instrum. 74, 895 (2003).

[23] O. L. Anderson, in Physical Acoustics, edited by W. P. Mason (Academic Press, New York, 1965), Vol. 3B, Chap. 2, pp. 43-95.

[24] CRC Handbook of Chemistry and Physics, edited by D. R. Lide (CRC Press, Boca Raton, Florida, 2001), 82nd ed.

[25] R. Vacher et al., J. Non-Cryst. Solids 45, 397 (1981).

[26] H. N. Lin et al., J. Appl. Phys. 69, 3816 (1991).

[27] V. E. Gusev et al., Laser Optoacoustics (AIP, New York, 1993).

[28] S. Adachi, J. Appl. Phys. 58, R1 (1985). 